\title{
Carbon paste electrodes modified by Pt and Pt-Ni microparticles dispersed in polyindole film for electrocatalytic oxidation of methanol
}

\author{
K.L. Nagashree, N.H. Raviraj, M.F. Ahmed* \\ Department of Studies in Chemistry, Central College Campus, Bangalore University, Bangalore 560 001, India
}

\section{A R T I C L E I N F O}

\section{Article history:}

Received 7 October 2009

Received in revised form

21 November 2009

Accepted 12 December 2009

Available online 22 December 2009

\section{Keywords:}

Electrooxidation

Methanol

Polyindole

Pt microparticles

Pt-Ni alloy

\begin{abstract}
A B S T R A C T
Electrocatalytic activity of the Pt microparticles dispersed into polyindole (PIn) films obtained by electropolymerization on carbon paste electrodes (CPE) towards methanol oxidation in perchloric acid medium has been demonstrated and investigated using cyclic voltammetry, potentiodynamic polarization, chronoamperometry and impedance spectroscopic techniques. The results show that PIn films obtained on these electrodes serve as good host matrices for the dispersion of Pt microparticles and exhibit good catalytic activity towards the electrooxidation of methanol compared to bulk Pt and CPE modified with Pt. The morphology and composition of the modified and unmodified films were obtained using SEM and EDX techniques. The effect of scan rate, amount of Pt and concentration of methanol, on the activity of the electrode has been tested. The effect of alloying Pt with different amounts of Ni was studied and it was found that when the metals are deposited from a solution containing the metal salts in $1: 1$ ratio the activity towards methanol oxidation was significantly enhanced.
\end{abstract}

(c) 2009 Elsevier Ltd. All rights reserved.

\section{Introduction}

Dispersion of Pt in very minute quantities in the form of microparticles inside a polymer matrix, a process generally referred to as polymer electrode modification leads to a remarkable enhancement in the electrocatalytic activity for the oxidation of methanol in its relevance to DMFCs, attributed essentially to (i) an increase in active surface area by dispersion of the catalyst and (ii) a better utilization of Pt crystallites in decreasing the poisoning effect of CO [1-3]. Such systems have not only been used for methanol oxidation but also for oxidation of other small organic molecules such as $\mathrm{HCHO}, \mathrm{HCOOH}$, ethylene glycol, and D-glucose [4,5]. Among the polymers that have found favour for use as host matrices for the development of such systems are mainly polyaniline, polypyrrole and polythiophene by virtue of their excellent conducting properties and very good adhesion to the substrate electrode. A number of other polymers have also been found to be conducive for such an application and they are poly(3,4-ethylenedioxythiophene) [6], poly(2-hydroxy-3-aminophenazine) [7], poly(o-aminophenol) [8], poly(o-phenylenediamine) [9], polyvinylpyridine [10], poly(4aminoquinaldine) [11] and poly(o-anisidine) [12]. The emphasis in all these studies has been on preparation and characterization of a support material in the form of a conducting polymer that is suitable for the dispersion of Pt and different alloys for electrocat-

\footnotetext{
* Corresponding author. Tel.: +91 80 22961346; fax: +91 8022961331.

E-mail address: mfahmed2007@rediffmail.com (M.F. Ahmed).
}

alytic purposes. All these have been successfully used for methanol oxidation reaction. The continuing efforts in this field show the possibility of using new polymer matrices for dispersion of catalytic particles and their use for electrocatalysing desired reactions. The work reported in this paper is therefore a continuation of the efforts at developing new conducting polymer matrices as support for catalytic dispersion. The work presented includes the development, characterization and modification of polyindole electrodes for methanol oxidation reaction.

Polyindole (PIn) though not as soughtafter as PANI or PPy for use as modified electrode due to its relatively low electrical conductivity, yet there are studies concerning the electropolymerization, characterization and use of this polymer as sensors and as anticorrosion coatings because of its good thermal and environmental stability [13-18]. In our study reported here therefore, we demonstrate the use of polyindole electrosynthesised from aqueous medium as a matrix for dispersion of metallic particles. We report the results of a study on the development of Pt, Pt-Ni/PIn modified electrodes and their use as electrocatalysts for the oxidation of methanol in acid medium under ambient conditions.

\section{Experimental}

All solutions were prepared using analytical grade reagents and double distilled water. The electrochemical experiments were performed using an Autolab PGSTAT30 potentiostat/galvanostat model with pilot integration controlled by GPES 4.9 software in a three-compartment cell (all interconnected) with a luggin capillary 
connecting the working and reference electrode chamber. Carbon paste electrode (CPE) with an area of $0.1256 \mathrm{~cm}^{2}$, Pt gauze and saturated calomel electrodes were used as working, counter and reference electrodes respectively. CPE was prepared by blending a mixture of graphite powder and paraffin through hand mixing and the paste was then inserted in a glass tube with electrical contact. A layer of the CPE was removed with a spatula and smoothened on an emery paper to get a smooth and fresh surface. The morphology of the as such and modified polymer films were examined using a scanning electron microscope (Leica Cambridge Ltd., Leica S 440i). The presence of catalytic microparticles in the polyindole matrix was confirmed by EDX data obtained from X-ray diffractometer (Oxford ISIS link software). The modified electrodes were then used as working electrodes for the electrooxidation of methanol in $\mathrm{HClO}_{4}$ medium. The electrocatalytic activity of the modified electrodes towards methanol oxidation was assessed on the basis of cyclic voltammetry, potentiodynamic polarization, chronoamperometry and electrochemical impedance spectroscopic studies. The relevant potential, frequency ranges and scan rates employed for these techniques are specified along with the results.

\section{Results and discussion}

\subsection{Electropolymerization of indole}

Reports on the electropolymerization of indole in aqueous solution are rare. Saraji and Bagheri [13] have studied the electropolymerization of indole in aqueous perchloric acid in detail and have optimized the conditions to obtain a highly electroactive polymer of indole on glassy carbon electrode. Based on these results, in all our studies reported here polymerization of indole was carried out however onto a clean CPE from a solution of $1 \mathrm{mM}$ indole in $1 \mathrm{M} \mathrm{HClO}_{4}$ by cycling the potential between -0.1 and $1 \mathrm{~V}$ at a scan rate of $25 \mathrm{mV} / \mathrm{s}$ for 10 cycles unless other wise mentioned. The corresponding cyclic voltammograms (CVs) (Fig. 1a) are very similar to those reported for indole by Saraji and Bagheri [13] in aqueous media and for other heterocyclic monomers like aniline and pyrrole in aqueous media. The first scan shows an irreversible anodic peak corresponding to the oxidation of indole at around $0.53 \mathrm{~V}$ vs. SCE. In the subsequent scans two pairs of anodic and cathodic peaks corresponding to different oxidation states of the product appear and the currents increase with the number of cycles. The polymerization process yielded a dark green film of polyindole on the substrate which represents doped state of the polymer. After polymerization the electrode was thoroughly rinsed with distilled water to remove any soluble oligomers and its electrochemical activity was studied in $1 \mathrm{M} \mathrm{HClO}_{4}$ in the potential range used for polymerization at different scan rates. The CVs as can be seen from inset of Fig. 1a are characterized by two pairs of anodic and cathodic peaks whose current values increase with scan rate and with continuous cycling showing that the polymer is electroactive in the chosen potential range. Talbi et al. [19] have attributed the first pair of peaks to the redox process pertaining to the polymer and second to doping-dedoping of polyindole by the anions of the electrolyte.

The PIn film synthesized for 10 cycles was not sufficient for recording IR spectrum and hence the electropolymerization was carried out at a constant potential of $0.8 \mathrm{~V}$ vs. SCE for about $10 \mathrm{~min}$ and the polymer was scraped off the electrode and collected. The IR spectrum of the so prepared PIn showed bands around 3430, 1633 and $1460 \mathrm{~cm}^{-1}$, the former being characteristic of elongation and deformation vibrations of $\mathrm{N}-\mathrm{H}$ indicating that the monomer units are not linked through the $\mathrm{N}$ of indole, and the latter two corresponding to stretching and bending modes of benzene ring which suggest that benzene rings are not involved in monomer coupling.
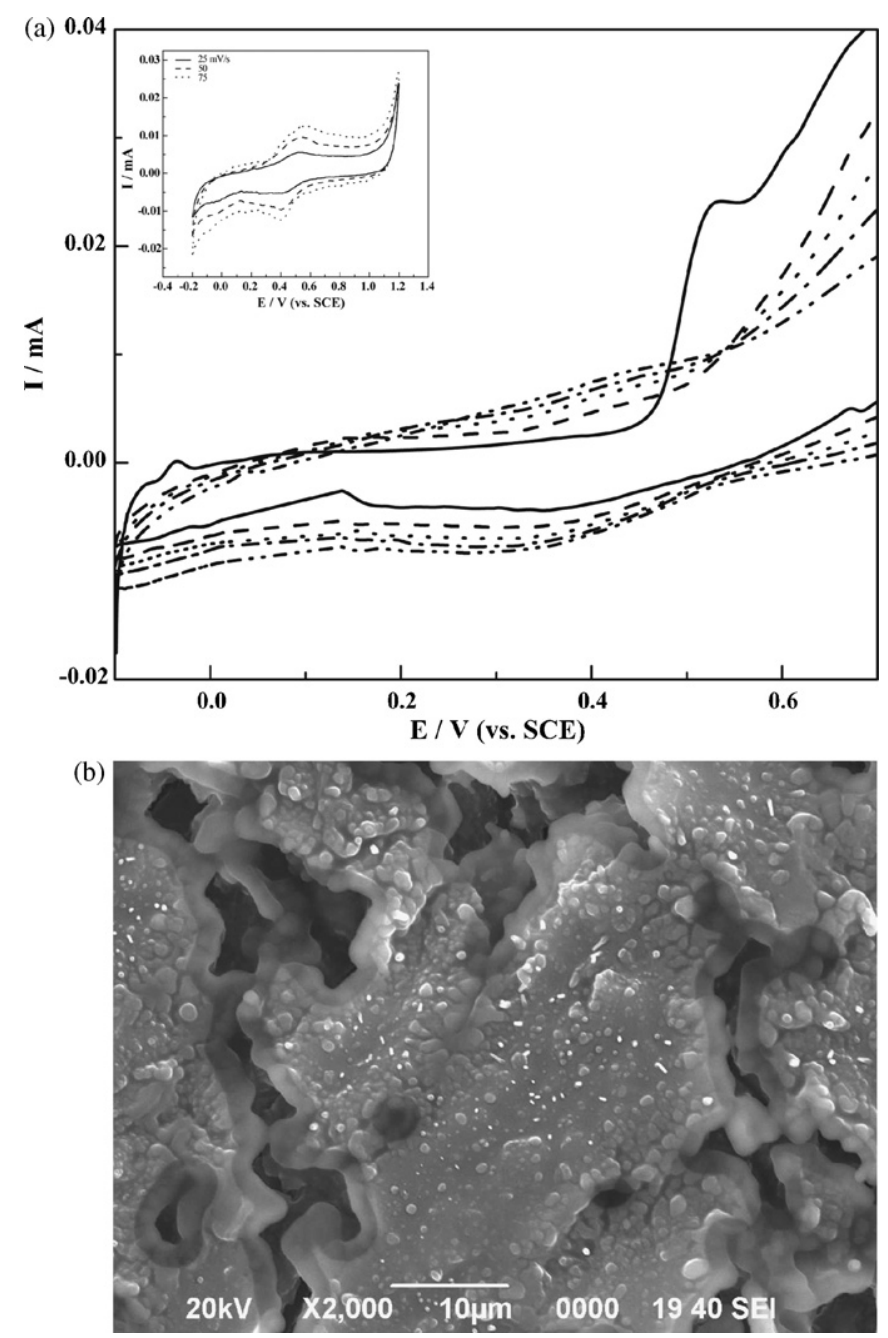

Fig. 1. (a) CVs recorded during electropolymerization of indole from a solution of $1 \mathrm{mM}$ indole in $1 \mathrm{M} \mathrm{HClO}_{4}$ at $25 \mathrm{mV} / \mathrm{s}$. Inset: Electrochemical behavior of PIn film in $1 \mathrm{M} \mathrm{HClO}_{4}$ at different scan rates. (b) SEM of Polyindole film on CPE.

Based on this data the formation of the polymer may be represented as in Scheme 1.

The SEM of PIn film on CPE (Fig. 1b) shows a scaly structure covered with nodules with deep crevices in between the scales. The presence of nodular structure beneath the crevices shows that the polymer is present in the form of layers to a certain extent and is covering the entire surface of the substrate.

\subsection{Modification of PIn films}

The PIn films obtained on CPEs were modified by incorporating Pt microparticles into the polymer matrix from $10 \mathrm{mM} \mathrm{K}_{2} \mathrm{PtCl}_{6}$ in $0.5 \mathrm{M} \mathrm{H}_{2} \mathrm{SO}_{4}$ potentiostatically at $-0.25 \mathrm{~V}$ vs. SCE until a charge corresponding to desired amount of Pt loading (assuming 100\% current efficiency for Pt reduction) was passed. The modification of the polymer was also brought about by carrying out deposition from solutions containing the salts of both $\mathrm{Pt}$ and Ni, i.e., $\mathrm{K}_{2} \mathrm{PtCl}_{6}$ and $\mathrm{NiSO}_{4}$ in various molar concentration ratios at $-0.25 \mathrm{~V}$ and the

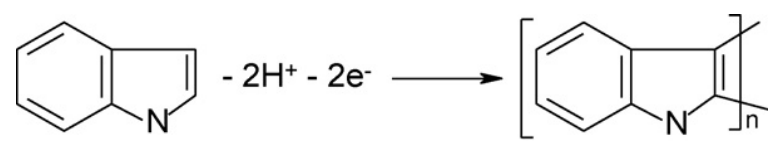

Scheme 1. Polymerization of indole. 
(a)

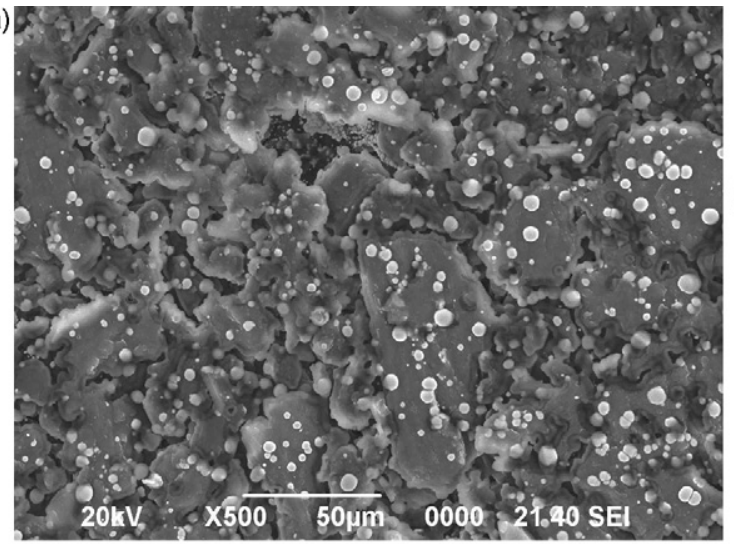

(b)

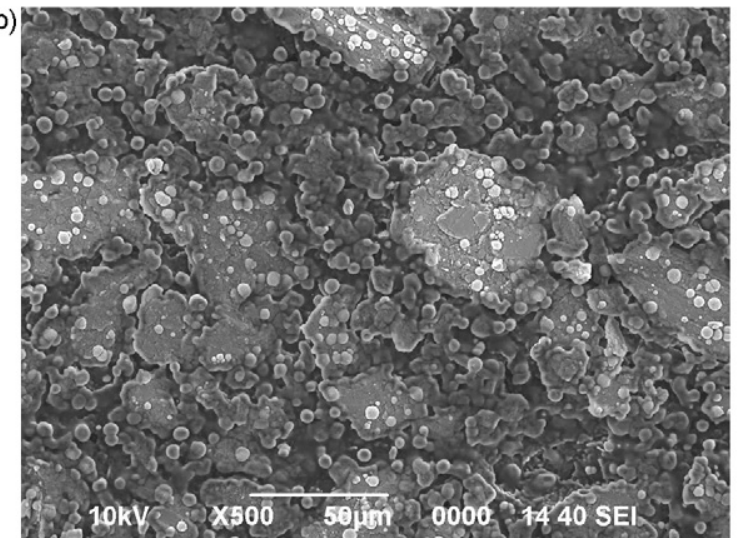

(c)

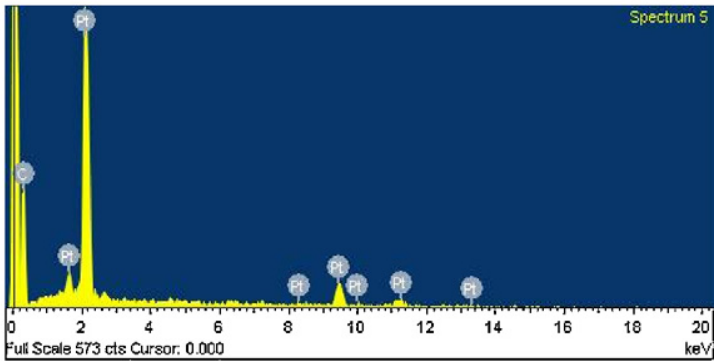

(d)

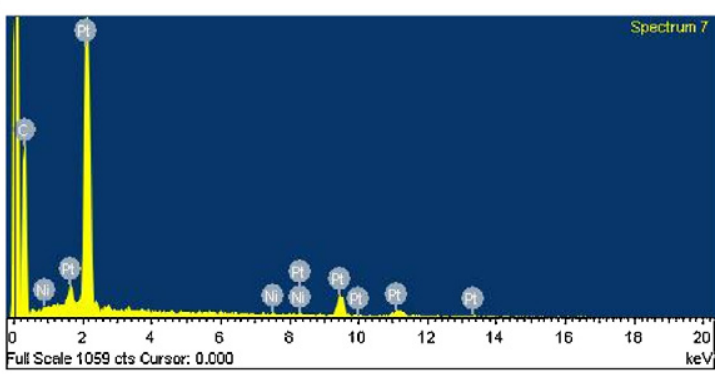

Fig. 2. SEM of (a) Pt modified PIn and (b) Pt-Ni modified PIn. EDX of (c) Pt modified PIn and (d) Pt-Ni modified PIn.

amount of charge passed in each case was kept constant. Fig. 2a and $b$ are the SEMs of the Pt and Pt-Ni (1:1) modified PIn films on two different CPEs while Fig. 2c and d shows the EDX spectra for the same.

\subsection{Electrooxidation of methanol on Pt modified polyindole substrates}

The electrocatalytic oxidation of methanol was carried out on bare CPE (CPE), bare Pt (Pt), PIn covered CPE (PIn/CPE), platinised $\mathrm{Pt}(\mathrm{Pt} / \mathrm{Pt}), \mathrm{Pt}$ modified CPE (Pt/CPE) and Pt modified PIn films on $\mathrm{CPE}$ (Pt/PIn/CPE) (the latter three of which contained the same amount of Pt, i.e., $0.1 \mathrm{mg}$ ) using cyclic voltammetry in the potential range $0-1 \mathrm{~V}$ at a scan rate of $25 \mathrm{mV} / \mathrm{s}$ in $0.5 \mathrm{M}$ methanol $+1 \mathrm{M}$ $\mathrm{HClO}_{4}$ solution. The inactivity of CPE towards methanol oxidation is in support of the observation that carbon electrodes do not catalyze methanol oxidation reaction in acid medium [20]. The PIn modified CPE also did not support methanol oxidation reaction at this concentration of methanol, but as the methanol concentration was increased the film started showing activity [21]. The currents were however insignificant and hence the corresponding CVs are not shown as they are hardly visible when they are superposed over those for Pt modified PIn film due to the large difference in the currents. In Fig. 3 the curve 'a' represents the CV recorded at the tenth potential scan for methanol oxidation on Pt/PIn/CPE. The cyclic voltammogram has characteristic peaks of methanol oxidation in the anodic and cathodic potential scans. The appearance of a pronounced anodic peak current in the cathodic potential scan is attributed to the removal of incompletely oxidized carbonaceous species formed in the forward scan [22]. These currents are essentially due to the dispersed Pt microparticles in the PIn matrix which catalyze the electrooxidation of methanol due firstly to the large increase in electrode surface area that the dispersal of Pt entails and secondly to the less sensitivity of Pt microparticles to poisoning by the adsorbed $\mathrm{CO}[3,23]$. The curve ' $\mathrm{b}$ ' in the figure is the CV for methanol oxidation on Pt/CPE. A comparison of the curves indicate a negative shift in the potential by a value of $\sim 35 \mathrm{mV}$ and a slightly higher current for methanol oxidation in presence of the polymer matrix attributed to the synergistic effect of the polymer matrix towards the dispersion of the Pt microparticles [24] and its effi-

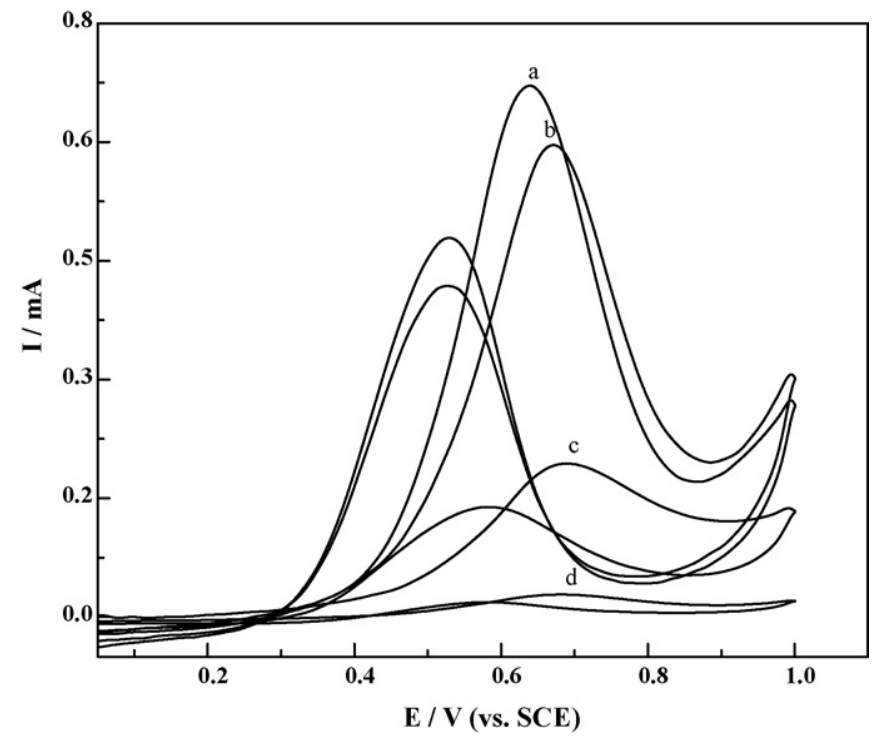

Fig. 3. CVs for oxidation of $0.5 \mathrm{M} \mathrm{CH}_{3} \mathrm{OH}$ in $1 \mathrm{M} \mathrm{HClO}_{4}$ at $25 \mathrm{mV} / \mathrm{s}$ on Pt/PIn/CPE (a), $\mathrm{Pt} / \mathrm{CPE}(\mathrm{b}), \mathrm{Pt} / \mathrm{Pt}$ (c) and bare Pt (d). 
cient utilization in catalyzing the reaction. The Pt/PIn/CPE shows an enhanced performance both in terms of potential and current compared to Pt/Pt (curve 'c') and Pt (curve 'd').

With repeated cycling, the catalytic currents remained almost constant for more than 50 cycles on Pt/PIn/CPE suggestive of the relative stability of the electrode for methanol oxidation. A few similar systems but with different polymer matrices, however, report a decrease in current for methanol oxidation in the first few scans and thereafter remaining constant during successive cycling $[6,8]$. The ratio of forward peak current to backward peak current which is a measure of efficiency of the electrode towards catalysis was found to be 1.4 which is quite a significant value [25]. The system being relatively new was examined in more detail to optimize conditions conducive for supporting better catalytic activity. The amount of Pt loaded into the polyindole film was kept constant at $0.1 \mathrm{mg}$ for all the studies unless otherwise mentioned.

In order to study the effect of thickness of the polymer film, the polymer film was deposited for different number of cycles in a solution of indole and perchloric acid. It was observed that the currents for methanol oxidation on thick modified PIn film decreased. This may be attributed to polymer film not having the right morphology for effective dispersal of catalytic particles. Thinner polymer films deposited for lesser number of cycles, after modification also exhibited small catalytic currents probably due to the incomplete growth of the polymer leading to inefficient dispersion of catalytic particles. The maximum catalytic currents were however observed on films modified after growing them for 10 cycles. Hence it can be concluded from the present study that, the polymer grown for 10 cycles has the right morphology for effective dispersal of catalytic microparticles and therefore gave the maximum currents for methanol oxidation.

The variation of methanol oxidation currents as a function of $\mathrm{Pt}$ loading on PIn films of constant thickness has been studied and it is evident from Fig. 4a that the methanol oxidation current increases with Pt loading and reaches a maximum value at a loading of $0.4 \mathrm{mg}$ in the PIn films. The increase may be attributed to the enhancement in the number of Pt microparticles with increase in loading. The currents, however, decline when the loading exceeds $0.4 \mathrm{mg}$ probably due to the agglomeration of Pt microparticles under heavier loading.

The methanol oxidation currents varied linearly with square root of scan rate indicating the involvement of diffusion controlled step in its oxidation (Fig. 4b). To support this, the oxidation of $0.5 \mathrm{M}$ methanol in $0.1 \mathrm{M} \mathrm{HClO}_{4}$ was carried out on a Pt rotating disc (diameter, $0.2 \mathrm{~cm}$ ) covered with PIn and modified with Pt at various rotation rates $(\omega)$ at $5 \mathrm{mV} / \mathrm{s}$. Koutecky-Levich plot $\left(I^{-1}\right.$ vs. $\omega^{-1 / 2}$ ) (Fig. 4c) obtained with this electrode for methanol oxidation at $0.6 \mathrm{~V}$ is linear, indicating that adsorption and diffusion of methanol through the PIn film is a controlling factor in its oxidation.

When the concentration of methanol was increased, the oxidation current increased upto to a methanol concentration of $0.8 \mathrm{M}$ after which it decreased (Fig. 4d) probably due to the saturation of catalytic sites by adsorption of methanol molecules.

The electrochemical impedance spectroscopic studies for the oxidation of methanol in perchloric acid medium on $0.1 \mathrm{mg} \mathrm{Pt}$ loaded PIn films was carried out at three different values of potential chosen from the foot and rising portion of the curve 'a' in Fig. 3, i.e., at $0.5,0.55$ and $0.6 \mathrm{~V}$ in the frequency range $10 \mathrm{KHz}$ to $50 \mathrm{mHz}$ with an amplitude of $5 \mathrm{mV}$ of the ac voltage employed. The plots at 0.5 and $0.55 \mathrm{~V}$ show characteristic semicircular behavior in high frequency region associated with charge-transfer process. However the plot at $0.6 \mathrm{~V}$, a potential close to the peak potential is highly scattered probably due to more than one mechanism prevailing for the oxidation of methanol through the formation of intermediates at this potential. A circuit having $R_{\mathrm{S}}$ in series with $R_{\mathrm{ct}}$ and CPE (in parallel) best fitted the experimental curve. Though the reac- tion is catalyzed at all the potentials chosen, the $R_{\mathrm{ct}}$ values directly obtained from the Nyquist plots show a least value at the potential close to the methanol oxidation peak. In Fig. 4e and its inset are the Nyquist and Bode plots obtained at $0.5 \mathrm{~V}$ for the oxidation of methanol with the solid lines representing the fitted curves.

Potentiodynamic polarization studies were carried out on $\mathrm{Pt}$ modified PIn film at various concentrations of methanol in $1 \mathrm{M}$ $\mathrm{HClO}_{4}$ at $1 \mathrm{mV} / \mathrm{s}$. At all the concentrations of methanol used for the study as is observed from Fig. $4 \mathrm{f}$ the oxidation of methanol starts around $0.35 \mathrm{~V}$ and the currents decrease after $0.5 \mathrm{~V}$. From the Tafel plot represented in the inset of Fig. $4 \mathrm{f}$ a value of $120 \mathrm{mV} / \mathrm{dec}$ was obtained as the Tafel slope indicating the involvement of one electron in the rate determining step of methanol oxidation reaction at this electrode.

Chronoamperograms recorded at this electrode at three different potentials are shown in Fig. 4g. The electrode showed good stability in terms of currents for a sufficiently good interval of time supporting the cyclic voltammetric data.

\subsection{Electrooxidation of methanol on Pt-Ni modified polyindole substrates}

Alloying of Pt group metals to enhance the performance towards electrooxidation of methanol has been carried out by many groups in different ways. The metals generally used to alloy $\mathrm{Pt}$ are $\mathrm{Ru}$ [26], Sn [27], Mo [28], Rh [29] and Ni [30]. These metals are also used in combinations to get ternary alloy systems [31,32]. In connection with polymer modified electrodes the enhancement is generally brought about by alloying Pt with either Ru or Sn. Swathirajan and Mikhail [2] investigated the effect of Pt-Sn catalysts electrodeposited on poly(3-methylthiophene) towards oxidation of methanol. Poly(2-hydroxy-3-aminophenazine) has been used as a matrix to disperse Pt, Pt-Sn and Pt-Ru particles and the alloy systems were reported to have higher activity towards methanol oxidation in perchloric acid medium than $\mathrm{Pt}$ alone [7]. Pt-Ru nanoparticles were deposited on polyaniline films and their catalytic activity was tested for methanol electrooxidation by Kessler and Castro Luna [33]. The catalytic activity of Pt particles towards methanol oxidation was found to be enhanced when Ru or Sn was co-deposited in poly(o-aminophenol) film [8]. These results are further supported by the work of Golikand et al. [9], who have also co-deposited Ru and Sn with Pt in poly(o-phenylenediamine) film and reported the enhancement of catalytic activity for methanol oxidation at these bi-metallic systems. Pt-Ru microparticles have been electrodeposited by pulse galvanostatic method onto nanofibrous polyaniline film and the electrodes showed considerably higher electrocatalytic activity for methanol oxidation [34]. The dispersion of Mo along with Pt and Ru in polyaniline matrix was found to reduce the sensitivity of the electrode system to $\mathrm{CO}$ poisoning as reported by Lima et al. [35]. Recently Galal et al. [36] have reported the use of electrodeposited Pt-Pd co-catalyst on poly(3methylthiophene) film for oxidation of methanol.

One however, hardly comes across studies involving the use of Ni to alloy with Pt within a polymer matrix for catalysis, the only instance in our knowledge being its use in a PANI matrix for electrocatalysis of CO [37]. It was, therefore, felt worthwhile to alloy Pt with $\mathrm{Ni}$ on polymer electrode and to carry out methanol oxidation on this bi-metallic polymer system. This system, however, poses a difficulty in that the potentials at which these two metals undergo reduction on the polymer differ to a large extent. This can be overcome by the use of a complexing agent. Alternately one can use a polymer which has free nitrogen that can easily complex with $\mathrm{Ni}$. It has been shown that when a conducting polymer matrix such as poly(1,5-diaminonaphthalene) or poly(o-aminophenol) is dipped in a $\mathrm{Ni}$ solution, $\mathrm{Ni}$ ions get complexed with the amine sites in the polymer backbone and these electrode systems have been suc- 

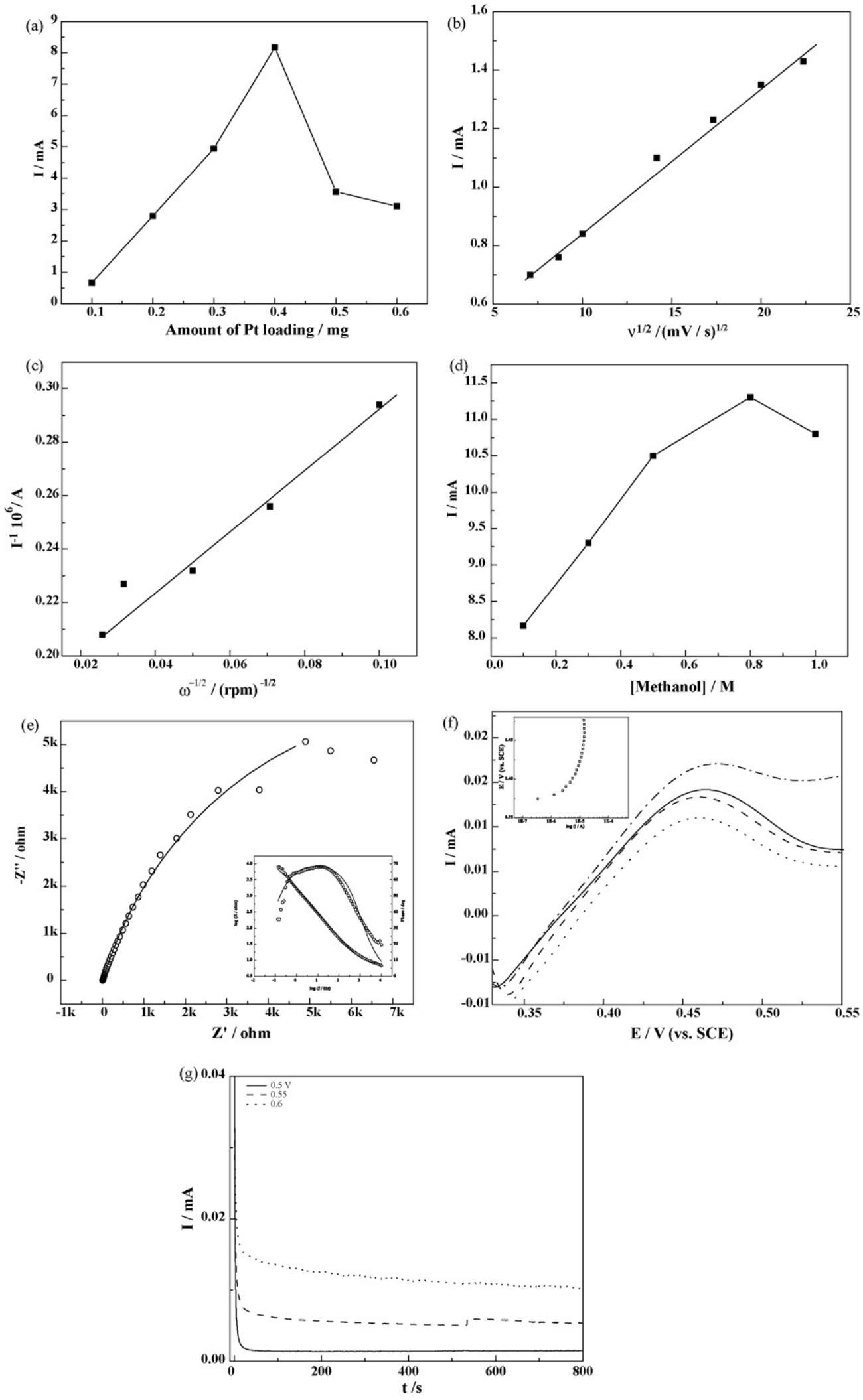

Fig. 4. (a) Oxidation currents of $0.5 \mathrm{M}$ methanol in $1 \mathrm{M} \mathrm{HClO}_{4}$ as a function of Pt loading on Pt/PIn/CPE film. (b) Variation of voltammetric anodic peak current for methanol oxidation as a function of square root of scan rate. (c) Koutecky-Levich plot for the oxidation of $0.5 \mathrm{M}$ methanol in $1 \mathrm{M} \mathrm{HClO}$ on Pt/PIn/Pt at $0.6 \mathrm{~V}$. (d) Oxidation currents of methanol in $\mathrm{HClO}_{4}$ as a function of methanol concentration on Pt/PIn/CPE film (Pt loading $0.4 \mathrm{mg}$ ). (e) Nyquist plot and Bode plot (inset) obtained at $0.5 \mathrm{~V}$ for the oxidation of methanol in $\mathrm{HClO}_{4}$ on Pt/PIn/CPE. Solid lines represent the fitted curves. (f) Potentiodynamic polarization plots obtained at $1 \mathrm{mV} / \mathrm{s}$ for different concentrations of methanol in $1 \mathrm{M} \mathrm{HClO}_{4}$ on Pt/PIn/CPE. Inset: Tafel plot for the oxidation of methanol. $(\mathrm{g})$ Chronoamperograms recorded at Pt/PIn/CPE at three different potentials in $0.5 \mathrm{M}$ methanol $+1 \mathrm{M}$ $\mathrm{HClO}_{4}$. 
Table 1

Bath composition for depositing different amounts of Pt and Ni on PIn film in $0.5 \mathrm{M}$ $\mathrm{H}_{2} \mathrm{SO}_{4}$ and the corresponding methanol oxidation currents obtained from $\mathrm{CV}$.

\begin{tabular}{lll}
\hline $\mathrm{Pt}(\mathrm{mM})$ & $\mathrm{Ni}(\mathrm{mM})$ & $\mathrm{I}(\mathrm{mA})$ \\
\hline 1 & - & 0.540 \\
1 & 0.5 & 3.26 \\
1 & 1 & 5.65 \\
1 & 2 & 2.98 \\
1 & 3 & 1.66 \\
1 & 4 & 1.77 \\
\hline
\end{tabular}

cessfully used to carry out methanol oxidation in alkaline medium [38,39].

In our study, baths containing Pt and $\mathrm{Ni}$ salts in different molar concentration ratios (Table 1) in $0.5 \mathrm{M} \mathrm{H}_{2} \mathrm{SO}_{4}$ were used and a potential $-0.25 \mathrm{~V}$ vs. SCE was applied to the PIn/CPE in order to obtain Pt-Ni incorporation into PIn film. Although this potential is adequate for reduction of $\mathrm{Pt}$, it is inadequate for the reduction of $\mathrm{Ni}$. Application of higher potentials required for reduction of $\mathrm{Ni}$ as well led to profuse evolution of hydrogen which rendered the polymer film unstable. Although we do not expect electrochemical incorporation of $\mathrm{Ni}$ in the polymer film along with $\mathrm{Pt}$ at this potential, it was found that Ni nevertheless gets incorporated into PIn film due essentially to its complexation with the free nitrogen in polyindole. The confirmation of the incorporation of $\mathrm{Ni}$ along with Pt is clearly evident from the EDX spectra (Fig. 2d) although the SEM showed no marked difference between the Pt modified PIn and Pt-Ni modified PIn (Fig. 2a and b). The amount of Ni incorporated is however much less than $\mathrm{Pt}$ as expected and the weight ratio of $\mathrm{Pt}$ to $\mathrm{Ni}$ obtained from EDX data was found to be 21.33:0.39. The electrodes obtained from these baths were then used to study the oxidation of methanol in $1 \mathrm{M} \mathrm{HClO}_{4}$ and the corresponding methanol oxidation currents are given in Table 1. The CVs for the oxidation of methanol carried out on PIn films modified by depositing the metals from solutions containing three different $\mathrm{Pt}$ and $\mathrm{Ni}$ salt ratios are given in Fig. 5a.

As can be seen from the oxidation current values in Table 1 and the CVs (Fig. 5a), the presence of $\mathrm{Ni}$ enhances the activity of $\mathrm{Pt}$ towards methanol oxidation. The maximum activity corresponding to an increase in current by 10 times that observed in the absence of $\mathrm{Ni}$ is shown by modified electrodes obtained from a solution containing equimolar amounts of $\mathrm{K}_{2} \mathrm{PtCl}_{6}$ and $\mathrm{NiSO}_{4}$. It is also observed from the cyclic voltammograms that the onset potential for methanol oxidation on the electrodes modified with $\mathrm{Ni}(0.3 \mathrm{~V})$ is lower than that observed in its absence $(0.4 \mathrm{~V})$. Though the ratio of forward peak current to backward peak current on these electrodes is less than 1 indicating poisoning of the electrode in the anodic scan by carbonaceous intermediates, the chronoamperograms recorded for methanol oxidation at the electrodes with and without Ni show no significant change in the percentage decrease in current (inset of Fig. 5a). The currents were constant during continuous cycling indicating limited dissolution of $\mathrm{Ni}$ from the deposit in the potential range used for methanol oxidation probably due to the stability of $\mathrm{Ni}$ in the polymer matrix and Pt lattice [40].

Various reasons have been given to explain the enhancement in the currents and reduction in the onset potential for methanol oxidation on Pt-Ni electrodes. It has been pointed out that the presence of various oxidation states of $\mathrm{Ni}$ in the deposit aid in improving methanol oxidation by providing oxygen species to Pt bonded CO and the metallic Ni helps to modify the electronic structure of Pt [40]. It has been reasoned out that increase in the d vacancy in the valence band $5 \mathrm{~d}$ orbital of Pt contributes to the improved electrocatalytic activity and durability of the Pt-Ni/Ni electrodes [41]. Abdel Rahim et al. [42] have suggested that the catalytic activity is enhanced due to increase in the number of active sites in the presence of $\mathrm{Ni}$, as well as through an electron donation process from $\mathrm{Ni}$
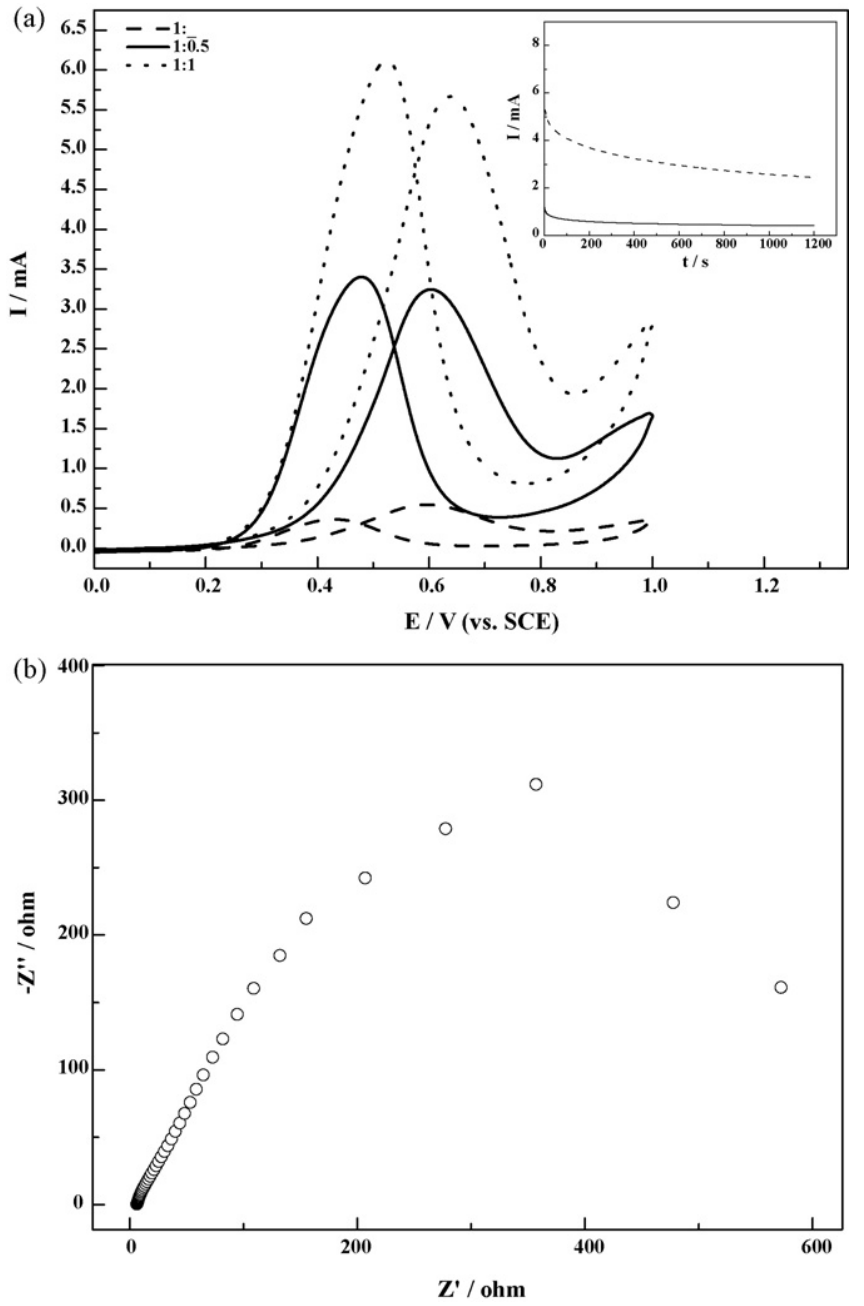

Fig. 5. (a) CVs for the oxidation of $0.5 \mathrm{M}$ methanol in $1 \mathrm{M} \mathrm{HClO}_{4}$ at $25 \mathrm{mV} / \mathrm{s}$ on $\mathrm{PIn}$ films modified by depositing Pt and Ni from solutions containing different concentration ratios. Inset: Chronoamperograms for the oxidation of $0.5 \mathrm{M}$ methanol in $1 \mathrm{M} \mathrm{HClO}_{4}$ on PIn films modified by depositing only Pt (solid line) and Pt-Ni (dashed line) from a solution containing their salts in the ratio $1: 1$. (b) Nyquist plot at $0.5 \mathrm{~V}$ for the oxidation of $0.5 \mathrm{M}$ methanol in $1 \mathrm{M} \mathrm{HClO}_{4}$ on PIn film modified by depositing $\mathrm{Pt}$ and Ni from a solution containing their salts in the ratio $1: 1$.

to Pt due to the transformation of $\mathrm{Ni}(\mathrm{OH})_{2}$ to $\mathrm{NiOOH}$. Enhancement in the activity of these electrodes may also be due to the increase in 'electroactive' platinum species formed by increase in defects in the Pt deposit due to introduction of Ni [43]. Therefore, with our electrode system we expect one or all of the above mechanisms to be influencing the activity of the Pt-Ni modified polyindole electrodes. However, more emphasis can be laid on the fact that nickel enhances the catalytic activity of platinum through an electron donation process that takes place once the $\mathrm{Ni}(\mathrm{OH})_{2} /(\mathrm{NiOOH})$ transformation begins, as the Nyquist plot for methanol oxidation at these electrodes show two semi-circles corresponding to two electrochemical reactions, i.e., for $\mathrm{Ni}$ and methanol oxidations (Fig. 5b).

\section{Conclusions}

The results indicate that PIn films can serve as good host matrices for polymer modified electrodes. The $0.1-0.4 \mathrm{mg}$ Pt modified PIn films obtained on CPEs show good catalytic activity towards oxidation of methanol as compared to Platinised CPE. Dependence of oxidation currents on methanol concentration and scan rate implies that the reaction is diffusion controlled at this electrode, 
and the Tafel slope value indicates the involvement of one electron in the rate determining step. From the amperometric and $\mathrm{CV}$ studies, the stability of the electrodes was demonstrated. The presence of Ni along with Pt enhances the performance of the PIn films towards oxidation of methanol. The maximum electrocatalytic activity towards oxidation of methanol is exhibited by PIn films modified from the bath containing the salts of Pt and $\mathrm{Ni}$ in the ratio $1: 1$.

\section{Acknowledgement}

This work was supported by UGC-DRS Programme (Phase-IV) in Chemistry, Bangalore University, Bangalore, India.

\section{References}

[1] H. Laborde, J.M. Leger, C. Lamy, F. Garnier, A. Yassar, J. Appl. Electrochem. 20 (1990) 524.

[2] S. Swathirajan, Y.M. Mikhail, J. Electrochem. Soc. 139 (1992) 2105.

[3] H. Laborde, J.M. Leger, C. Lamy, J. Appl. Electrochem. 245 (1994) 219.

[4] F. Ficicioglu, F. Kadirgam, J. Electroanal. Chem. 451 (1998) 95.

[5] I. Becerik, F. Kadirgan, J. Electroanal. Chem. 436 (1997) 189.

[6] S. Patra, N. Munichandraiah, Langmuir 25 (2009) 1732.

[7] A. Kelaidopoulou, E. Abelidou, G. Kokkinidis, J. Appl. Electrochem. 29 (1999) 1255.

[8] S.M. Golabi, A. Nozad, Electroanalysis 15 (2003) 278.

[9] A.N. Golikand, S.M. Golabi, M.G. Maragheh, L. Irannejad, J. Power Sources 145 (2005) 116.

[10] Yu.M. Maksimov, O.V. Afanas'eva, B.I. Podlovchenko, Russ. J. Electrochem. 31 (1995) 133.

[11] A.A. Hathoot, M. Abdel Kader, M. Abdel Azzem, Int. J. Electrochem. Sci. 4 (2009) 208.

[12] C. Sivakumar, Electrochim. Acta 52 (2007) 4182.

[13] M. Saraji, A. Bagheri, Synth. Metals 98 (1998) 57.

[14] P.C. Pandey, R. Prakash, J. Electrochem. Soc. 145 (1998) 4103.

[15] P.C. Pandey, Sens. Actuators B: Chem. B54 (1999) 210.
[16] R. Prakash, R.C. Srivastava, P.C. Pandey, J. Solid State Electrochem. 6 (2002) 203.

[17] T. Tuken, B. Yazici, M. Erbil, Surf. Coat. Technnol. 200 (2006) 4802.

[18] M. Dudukcu, F. Koleli, Progr. Org. Coat. 55 (2006) 324.

[19] H. Talbi, B. Humbert, D. Billaud, Synth. Metals 84 (1997) 875.

[20] A. Wieckowski, in: J.O'M. Bockris, B.E. Conway, R.E. White (Eds.), Modern Aspects of Electrochemistry, vol. 21, Plenum Press, New York, 1990.

[21] K. Rajendra Prasad, N. Munichandraiah, J. Power Sources 103 (2002) 300.

[22] R. Manohara, J.B. Goodenough, J. Mater. Chem. 2 (1992) 875.

[23] P.O. Esteban, J.M. Leger, C. Lamy, F.C. Garnier, A. Yassar, J. Appl. Electrochem. 20 (1990) 520

[24] A. Lenoe, W. Marino, B.R. Scharifker, J. Electrochem. Soc. 139 (1992) 438.

[25] Z. Liu, X.Y. Ling, X. Su, J.Y. Lee, J. Phys. Chem. B 108 (2004) 8234.

[26] N.Jha, A. Leela Mohana Reddy, M.M. Shaijumon, N. Rajalakshmi, S. Ramaprabhu, Int. J. Hydrogen Energy 33 (2008) 427.

[27] E.E. Switzer, T.S. Olson, A.K. Datye, P. Atanassov, M.R. Hibbs, C.J. Cornelius, Electrochim. Acta 54 (2009) 989.

[28] S. Mylswamy, C.Y. Wang, R.S. Liu, J.F. Lee, M.J. Tang, J.J. Lee, B.J. Weng, Chem. Phys. Lett. 412 (2005) 444.

[29] R.T.S. Oliveira, M.C. Santos, P.A.P. Nascente, L.O.S. Bulhoes, E.C. Pereira, Int. J. Electrochem. Sci. 3 (2008) 970.

[30] Y. Zhao, E. Yifeng, F. Louzhen, Y. Qiu, S. Yang, Electrochim. Acta 52 (2007) 5873.

[31] X. Zhang, F. Zhang, R.F. Guan, K.Y. Chan, Mater. Res. Bull. 42 (2007) 327.

[32] T.Y. Morante Catacora, Y. Ishikawa, C.R. Cabrera, J. Electroanal. Chem. 621 (2008) 103.

[33] T. Kessler, A.M. Castro Luna, J. Appl. Electrochem. 32 (2002) 825.

[34] H.H. Zhou, X.H. Ning, J.H. Chen, W.Z. Wei, Y.F. Kuang, Ind. J. Chem. A 44A (2005) 1009.

[35] A. Lima, F. Hahn, J.M. Leger, Russ. J. Electrochem. 40 (2004) 326.

[36] A. Galal, N.F. Atta, S.A. Darwish, S.M. Ali, Top. Catal. 47 (2008) 73.

[37] G.P. Jin, X. Peng, Y.F. Ding, W.Q. Liu, J.M. Ye, J. Solid State Electrochem. 13 (2009) 967.

[38] R. Ojani, J.B. Raoof, S.R.H. Zawarmahalleh, Electrochim. Acta 53 (2008) 2402.

[39] R. Ojani, J.B. Raoof, S. Fathi, J. Solid State Electrochem. 13 (2009) 927.

[40] J. Mathiyarasu, A.M. Remona, A. Mani, K.L.N. Phani, V. Yegnaraman, J. Solid State Electrochem. 8 (2004) 968.

[41] T. Nakashima, S. Nohara, H. Inoue, C. Iwakura, Res. Chem. Interm. 32 (2006) 561.

[42] M.A. Abdel Rahim, H.B. Hassan, R.M. Abdel Hameed, Fuel Cells 7 (2007) 298.

[43] T.C. Wen, C.H. Hu, C.C. Hu, J. Chin. Inst. Chem. Eng. 30 (1999) 515. 